\title{
Les centres commerciaux, cibles d'incivilités et promoteurs de
} sécurité

In: Déviance et société. 1993 - Vol. 17 - N³. pp. 235-260.

Citer ce document / Cite this document :

Ocqueteau Frédéric. Les centres commerciaux, cibles d'incivilités et promoteurs de sécurité. In: Déviance et société. 1993 - Vol. 17 - N³. pp. 235-260.

doi : $10.3406 /$ ds. 1993.1305

http://www.persee.fr/web/revues/home/prescript/article/ds_0378-7931_1993_num_17_3_1305 


\section{Abstract}

This paper describes an original private-policing experiment, initiated in a socio-cultural context favouring community policing, by a shooping mall in a so- called hard neighborhood. This experiment challenges many generally accepted ideas about prevention of incivilities in a privately owned space open to the public. A drop in the level of losses incurred by the supermarket cannot be the unique criterion by which situational prevention's efficiency can be evaluated. Specific community prevention programs, focusing on youngsters and on the perceived causes of their idleness and their incivilities should also be taken into account. This approach rocks the balance of ordinary division of labour relative to social control. Although a long term evaluation of the experiment's success remains a difficult task (which would be the criteria?), it still leaves room for a new approach of shopping malls in France as more than organized victims struggling to defend their premises; they can be viewed as possible partners in the general production of urban safety.

\section{Résumé}

Dans une conjoncture socio-politique favorable à l'institutionnalisation d'expériences de «polices de proximité», la présente monographie décrit une expérience de gardiennage originale, initiée par un centre commercial implanté dans une cité de banlieue dite à problèmes. L'expérience remet en cause bien des idées reçues concernant la prévention des incivilités sur un espace privé ouvert au public. Car le critère d'efficacité au principe de l'obligation de résultat des mesures de prévention situationnelle, ne réside pas seulement dans le constat de la diminution des pertes subies par le supermarché. II réside aussi dans des actions spécifiques centrées sur les causes perçues du désœuvrement et incivilités commises par les jeunes, des actions de prévention communautaire et sociale entreprises à leur égard. Cela remet en cause l'équilibre sur lequel repose habituellement la division sociale du travail de régulation de l'ordre. Si une évalua- tion à long terme de la réussite de l'expérience reste difficile à tenter (quels en seraient les critères?), on peut néanmoins comprendre pourquoi des centres commerciaux commencent à apparaître en France, non plus seulement comme des victimes organisées pratiquant comme elles le peuvent la défense de leur site, mais encore comme de possibles partenaires dans la production générale de la sécurité urbaine.

\section{Zusammenfassung}

Vor dem Hintergrund eines der Idee eine «burgernahen Polizei» fôrderlichen sozio-politischen Klimas beschreibt die vorliegende Untersuchung Erfahrungen, die mit einem durch ein Geschàftszentrum initierten Überwachungsmodell gemacht wurden, das in einem als «sozialer Brennpunkt» bezeichneten Vorort errichtet worden war. Die Erfahrungen lassen an den bislang zum Bereich der Erhaltung von Ordnung in einem privaten Raum, der der Öffentlichkeit zugänglich ist, enwickeiten Vorstellungen zweifeln. Denn das Effizienzkriterium, das im Zusammenhang mit Präventionsmaßnahmen Verwendung finden muß, erfaßt nicht nur die Feststellung der Minimierung von materiellen Verlusten, die die Geschäfte erleiden. Das Effizienzkriterium bezieht sich gleichermaBen auf die spezifischen Aktionen, die sich auf die perzipierten Ursachen des Herumlungerns von Jugendlichen und von ihnen hinterlassener Unordnung sowie kommunale und soziale Präventionsmaßnahmen, die sich auf diese Probleme beziehen, konzentrieren. Dies stellt das Gleichgewicht in Frage, auf dem normalerweise die Regulierung der Ordnung beruht. Wenn auch eine externe Evaluation des Erfolgs derartiger Experimente schwierig bleibt (anhand welcher Kriterien sollte der Erfolg auch gemessen werden ?), so kann man nichts- destotrotz verstehen, warum Geschäftszentren heutzutage in Frankreich nicht mehr lediglich als Opfer erscheinen, die sich, so gut es geht, verteidigen, sondera als potentielle Partner in der Herstellung von Sicherheit in den Städten.

In een socio-politiek klimaat dat blijkbaar positief staat tegenover de institu- tionalisering van de ervaringen met lokale politie (community policing) ter voor-koming van delikwentie en inbreuken op de openbare orde, beschrijft deze monografîe een origineel experiment in de «bewakingsbeveiligingssfeer» dat werd opgestart op initiatief van een winkelcentrum un een probleemwijk van een Parijse voorstad. Het experiment gaat in tegen aile bestaande ideeën omtrent ordehandhaving in «voor het publiek tocgankelijke private plaatsen». De doel- matigheid, hier met betrekking tot de resultaten 
van preventieve maatregelen, wordt immers niet alleen afgemeten aan de vermindenng van de kosten die door het winkelcentrum worden geleden, maar evenzeer aan de specifieke akties die toegespitst worden op de oorzaken van de jeugdwerkloosheid en verveling zoals ze gepercipieerd worden door de promotoren van het experiment. Door dit ailes wordt een groot vraagteken geplaatst bij het evenwicht waarop «het welzijns- werk in het kader van orderegulering» berust. Zelfs als een externe evaluatie van het welslagen van een dergelijk experiment moeilijk blijkt (welke kriteria zou men ervoor moeten hanteren?), verhindert dit niet dat men kan begrijpen waa- rom winkelcentra zich niet alleen beginnen te profileren als georganiseerde slachtoffers die hun eigen ruimten beginnen te verdedigen maar ook als legi- tieme partners en co-producenten van stedelijke veiligheidszorg. 


\title{
LES CENTRES COMMERCIAUX, CIBLES D'INCIVILITÉS ET PROMOTEURS DE SÉCURITÉ
}

\author{
F. OCQUETEAU*
}

Face aux flambées de violences qui gagnent de temps à autre certains quartiers de banlieues françaises (en 1981, 1990 et 1991 notamment), face aux déchirures du tissu social dont elles témoignent, les questions de sécurité se posent aujourd'hui avec une acuité décuplée. Or, si l'on constate bien une prolifération de littératures diagnostiques à l'égard des insécurités liées au mal des banlieues ou aux quartiers périphériques, rarissimes demeurent encore les évaluations centrées sur l'art et la manière dont y sont pratiqués non seulement le maintien de l'ordre, mais encore et surtout la sécurité au quotidien.

Si l'on souhaite rabattre l'insécurité aux problèmes des délinquances, le cadre analytique de Lagrange (1993) paraît à l'heure actuelle le plus prometteur qui fait un sort définitif à la conséquence généralement tirée de la célèbre prémisse de Durkheim: si la collectivité renforce ce qui l'unit dans la réprobation du crime, on ne saurait en déduire pour autant que le crime renforcerait la cohésion sociale. Au contraire, le crime pourrait faire s'effondrer une communauté si les structures et forces qui l'unissaient préalablement étaient sur la voie de se déliter : soit par effet d'un affaiblissement du contrôle social endogène à la communauté d'appartenance (qui pourrait expliquer l'augmentation du taux des crimes d'appropriation par certains de ses membres, dans les quartiers riches); soit, par effet d'un affaiblissement du contrôle social exogène à la communauté, lié au cumul d'une multitude de handicaps sociaux vouant progressivement des populations entières à la précarité, sinon à la relégation (Delarue, 1992). Ces situations d'impasse sociale conduiraient à la montée de violences sur les lieux mêmes de l'exclusion, et les processus d'exclusion renforceraient indéfiniment la spirale de la ségrégation sociale.

Dans pareil contexte, l'action répressive des forces de l'ordre serait peu probante à moyen terme, parce que le retour à la sécurité (qui passe surtout par des actions de prévention des incivilités $\left.{ }^{1}\right)$, condition majeure à la préservation de l'identité communautaire, s'accommode assez mal de solutions d'autant plus bru-

\footnotetext{
CNRS/CESDIP, Paris.
}

1 Au sens général et particulier donné par Roché (1993) à ce terme : comportements à la limite de la légalité et pas toujours nécessairement incriminés par la loi pénale; menaces des rituels sociaux à l'aide desquels est évaluée l'innocuité du rapport à autrui. 
tales qu'elles demeureraient épisodiques, en tout cas, sans grand effet sur lesdites incivilités. Or, faire diminuer l'insécurité passe pour une très large part par la prévention micro-sociale des incivilités quotidiennes éprouvant durement certains quartiers urbains.

Nous savons que sécurité personnelle des citoyens et bonne gestion de l'identité collective vont de pair, les civilités constitutives de la citoyenneté ordinaire fondant l'appartenance à la cité (Roché, 1993, 293). Nous savons également que des logiques socio-politiques persistant de longue date à moins tenir compte de l'habitat social qu'à tout miser sur l'accession à la propriété ont conduit à l'existence de quartiers $\grave{a}$ problèmes dans certaines banlieues francaises, même si elles ne sont en rien comparables à celles ayant historiquement conduit à la constitution des ghettos à Chicago (Wacquant, 1992, 1993). Le «lien social» n'est pas à ce point attaqué et détruit que les actions de prévention sur les incivilités dans ces quartiers seraient vaines. Toutefois, pourra-t-on longtemps soutenir que le modèle de prévention de la délinquance mis en oeuvre en France depuis une dizaine d'années, et souvent considéré comme le modèle alternatif à la situationrepoussoir américaine, serait réellement efficace?... Il ne fait pas de doute qu'on en reste encore trop souvent à la proclamation d'intention, tandis que les évaluations des retombées pratiques des politiques impulsées par le haut demeurent toujours rares, ou du moins fort lacunaires (Robert, Renouard, 1991, 202). Ne se pourrait-il pas également que du côté de l'analyse critique, toute évaluation d'actions de sécurisation soit inhibée par le risque de bousculer pas mal de conformismes intellectuels pouvant remettre en cause les frontières les mieux établies dans les habituelles façons de penser les phénomènes d'ordre et de désordres? Nous voulons parler de ces couples antithétiques qui ne reflètent bien souvent que les découpages administratifs de l'action des divers spécialistes de l'intervention sociale: prévention vs répression; autorités centralisées vs autorités décentralisées; travailleurs sociaux $v s$ policiers, etc. Le diagnostic de l'observateur sociologue pourrait aider à dépasser de tels clivages sans céder à sa mission critique. Face à des pratiques inventives lui paraissant dénouer des situations sociales apparemment bloquées, que lui faut-il penser ? Notamment de pratiques jouant sur les trois grands registres d'action disponibles : prévention sociale, prévention communautaire et prévention situationnelle (Hastings, 1993)? Peut-il d'emblée, et sans y regarder à deux fois, ranger dans une catégorie ou l'autre et laquelle, l'action d'acteurs, ni totalement policiers, ni totalement travailleurs sociaux, culturellement proches des populations à problèmes qu'ils contrôlent, les aidant à s'intégrer, tout en continuant à répondre au mandat particulier pour lequel ils sont payés : démontrer à leur commanditaire (l'enseigne principale d'un hypermarché autour duquel s'agrègent de petites unités de commerces par exemple), qu'ils peuvent faire baisser rapidement certains niveaux d'insécurité liés aux actes illicites de gens issus du site où ils interviennent?...

Par delà ce genre de questions, on doit se demander au fond si le mal des banlieues serait devenu à ce point profond et désespéré que toute innovation microsociale serait fatalement vouée à l'échec. Nous n'en croyons rien. Voilà pour- 
quoi, à travers une réflexion liminaire, nous poserons quelques-uns des jalons théoriques indispensables à la possibilité d'une analyse critique de l'expérimentation sociale des pratiques de sécurité (I). A la suite de quoi, nous présenterons les résultats principaux d'une monographie basée sur une enquête de terrain : on tentera d'y montrer comment le chef de sécurité d'un hypermarché et son service de sécurité sont apparemment parvenus à durablement pacifier des rapports sociaux envenimés dans un quartier de banlieue dégradé où il est implanté. On aura dâ prendre au préalable connaissance du contexte agité au sein duquel a pu s'inscrire l'expérience, de façon à en montrer la singularité et les raisons (II). Une conclusion plus prospective nous la fera relativiser.

\section{Pour une sociologie des pratiques de sécurité innovantes}

Rechercher un meilleur état de sécurité dans certains quartiers de banlieue passe évidemment par des actions sur les structures exogènes, ce qui demeure pour une bonne part du domaine de l'action de longue haleine des politiques publiques. Nous entendons par là les mesures de prévention sociale visant à agir sur les causes premières des actes délictueux : mésententes familiales, chômage, échec scolaire, etc. Mais également, des mesures de prévention communautaire, c'est-à-dire les actions de collectivités visant à faire reculer les conduites délictueuses en améliorant le cadre de vie, l'habitat, et en développant la vie collective. Qui plus est, croire en la possible efficacité du combat quotidien contre le sentiment d'insécurité lié aux diverses incivilités, par le biais d'une prévention situationnelle de complément, n'est pas forcément l'expression d'un idéalisme ou d'un volontarisme naîfs. Aucune action innovante n'est possible si l'on ne croit pas un tant soit peu à l'homme ou du moins aux capacités que pourrait avoir, chacun à son niveau, de vouloir prendre en charge une part du destin collectif de la communauté de vie dans le quartier.

Deux exemples d'analyse puisés dans des sociologies critiques d'inspirations différentes, mais communément sensibles au vécu d'agents sociaux spécialisés ou non dans des fonctions de sécurité formelles ou informelles, peuvent nous en convaincre. Un troisième exemple, puisé quant à lui, dans le domaine de la réflexion technocratique d'Etat à propos des polices dans la ville, devrait nous permettre ensuite d'articuler le propos à la présentation de la monographie.

1. Il apparaît par exemple que les explorations en profondeur issues des entretiens menés par l'équipe de Pierre Bourdieu (Bourdieu, 1993) avec des locataires de cités $\mathrm{HLM}^{2}$ nuancent fortement la critique convenue d'un membre de la même équipe, à propos de ce qu'il désigne comme le traitement médiatico-politique du problème des banlieues (sic) (p. 78). Car si cette socio-

2 Habitation à loyer modéré: une forme d'habitat social. 
logie spécialisée dans l'étude des effets de la domination sociale nous apprend en définitive que les agents sociaux n'ont pas nécessairement accès au principe de leur mécontentement ou de leur malaise (pp. 918-919), et que si le sociologue peut les aider à la façon d'un accoucheur (p. 919), alors on est en droit de tirer la conclusion qu'il existe une voie d'action possible pour les dominés, située entre la révolte et le désespoir, et cela, à la lecture même des entretiens en question. Concernant les habitants, on voit une commerçante refuser de rendre les autres responsables de son malheur (p. 117), une habitante d'origine portugaise chercher à s'améliorer sans vouloir l'impossible (p. 107), un retraité engagé dans une entreprise volontariste de rééducation des habitants pour renouer avec l'image de la solidarité ouvrière de jadis (p. 124), un collectif d'habitants protester contre le mépris journalistique dont ils se sentent l'objet (p. 133), l'épouse d'un gardien d'immeuble, indulgente à l'égard des enfants en général, éprouver avec son époux la contradiction de qui pourrait presque excuser des comportements tenus par ailleurs pour moralement inacceptables (p. 141). Concernant divers agents du travail social, on voit le directeur d'une mission locale faire de l'occupationnel, mais ne pas se résoudre à ce que son investissement militant se laisse engloutir par les logiques induites par son statut de fonctionnaire (p. 258); le chef d'un projet $\mathrm{DSQ}^{3}$ défendre la bureaucratie contre elle-même en investissant toute son énergie dans une mission à laquelle elle croit, bien qu'emprisonnée dans sa fonction (p. 231); le principal du collège d'une Zone d'Education Prioritaire assumer la violence dans son établissement sans la dramatiser, à force de trouver des astuces de discipline (p. 683), etc.

Bref, si la misère de position est un phénomène perceptible à l'investigation sociologique, maints indices confortent le lecteur dans l'idée qu'il existerait parmi ceux qui souffrent d'un mal-être social, au moins à l'état latent, des ressources les prédisposant à résister individuellement ou collectivement, de la place même où ils sont situés, à la condition qu'ils subissent. Cela semble vouloir dire que peuvent leur être redonnés des motifs d'espérer, venus tant de l'action politique que de l'action sociologique.

2. Plus spécifiquement, Dulong et Paperman (1992) montrent, à partir de leur démarche ethnométhodologique, en quoi les problèmes de sécurité fonctionnent comme une solution organisationnelle aux problèmes de la (mauvaise) réputation du quartier. En effet, évoquer l'insécurité du lieu où ils vivent est une opération vouée à demeurer à l'état d'implicite tant que des habitants, voire les gestionnaires de la cité HLM, n'ont pas de raisons particulières pour donner à ce syntagme un contenu méthodique, organisé, et détaillé. En revanche, pratiquer des actions de mise en sécurité fait partie d'une opération dicible permettant de n'avoir pas à désigner, à stigmatiser la situation du site où l'on vit... Bref, agir

3 Développement social des quartiers: un programme de la politique de la ville. 
pour sécuriser autorise à se donner un objectif, des moyens pour l'atteindre, et permet d'en rendre compte. Pour le gestionnaire de la cité HLM par exemple, il y va de la possibilité socialement reconnue et donc publiquement mentionnable d'une action organisée, légitime et valorisée (p. 141). Notons qu'on n'est plus dans la logique de la dénonciation de l'insécurité : on est passé au contraire à celle de l'action pour la sécurité, de façon à freiner les processus de destructuration sociale. Quel que soit le statut de ceux qui sont engagés dans de telles actions, ils expérimentent en somme spontanément la célèbre théorie de Wilson et Kelling (1982) selon laquelle toute fenêtre cassée non immédiatement réparée, serait une invincible incitation au pillage, à la dégradation, à la marginalisation du quartier.

3. Après douze années de politique de prévention de la délinquance, il semble que la délégation interministérielle à la ville (Linhart, 1993) réoriente désormais sa réflexion à partir des leçons du bilan qu'elle tire des actions passées (CNV, 1992), lesquelles sont loin comme on s'en doute, d'avoir toutes été couronnées de succès. On se plaît à découvrir désormais $(C N V, 1993)$ que beaucoup de métiers ayant à voir de près ou de loin avec la sécurité auraient largement commencé d'évoluer vers des actions dites de proximité. Non seulement celles des policiers d'Etat, des policiers municipaux, ou celles des enseignants opérant dans les zones sensibles, mais également celles des régisseurs du logement social. Non seulement, celles des conducteurs et receveurs de transports, mais encore celles des vigiles et autres agents de surveillance. Bref, police de proximité par îlotage, expériences de médiation sociale, sécurité privée, etc. seraient des concrétisations d'actions rendues possibles à cause de la place occupée par des agences de contrôle social de statut multiple, publiques, semipubliques, privées, mais toutes peu ou prou engagées dans la promotion d'actions de sécurité sur des segments d'espaces urbains connaissant des vulnérabilités spécifiques. Sont passés au crible de l'analyse: le logement social, l'école, les transports publics, et les centres commerciaux, pour autant qu'ils sont perçus comme des espaces vecteurs où se manifeste le besoin d'y co-produire et coordonner des actions de sécurité.

On observera que c'est la première fois qu'en France un tel document expose une philosophie d'action pour les centres commerciaux, promus partenaires légitimes dans le domaine de la prévention de la délinquance, et vus comme des acteurs essentiels du développement social urbain par leur insertion dans le tissu social des banlieues. Ce document reconnaît que vols à l'étalage, vols à l'arraché commis sur clients, délinquances sur les parkings, présence de marginaux, problèmes liés à la drogue y sont autant de causes d'insécurité susceptibles de dégénérer en incidents graves. Aussi, l'utilisation intensive des agents privés de sécurité est-elle reconnue comme légitime, à la condition que soit préalablement revalorisé leur métier, et que leur statut soit mieux rationalisé par le droit. De multiples recommandations y sont par ailleurs exposées concernant non seulement la revalorisation du statut des agents de surveillance, mais encore les condi- 
tions dans lesquelles les chaînes de la grande distribution pourraient être incitées à s'engager dans des actions de prévention au delà de la seule défense de leur site et clientèle.

Nous reviendrons in fine sur les conséquences qu'il convient de tirer de l'ensemble de ces nouvelles logiques partenariales, à la lumière de la présentation de notre monographie. Reconnaissons pour l'instant que ces prises de position sociologiques ou technocratiques contribuent paradoxalement à débloquer une pensée «préventionniste», laquelle est en général entravée par deux phénomènes inhibiteurs assez classiques :

- ou bien, la peur d'avoir à légitimer des options politiques conservatistes (law and order). D'aucuns en effet n'ont pas manqué d'argumenter a la fois sur deux terrains pour eux indissociables : un Etat cherchant à dissuader des comportements déviants par l'exemplarité d'une répression féroce des atteintes aux personnes, et une société civile qui se protègerait des atteintes aux biens en recourant aux ressources du commerce.

- ou bien, la mauvaise conscience engendrée par les conséquences souvent irréalistes qu'il conviendrait de tirer d'options radicales émises à propos d'une société conflictuelle, dont le crime ne serait qu'un symptôme de la crise, mais contre lequel le commerce de la sécurité viendrait relayer des pouvoirs publics impuissants à enrayer eux-mêmes les effets de cette crise.

La pensée préventionniste guidant l'action sociale ne peut être que molle : elle est forcément située entre ces deux extrêmes.

\section{Une ótude de cas}

La présente monographie donne le coup d'envoi à une recherche non spécifiquement commanditée, constituée d'une dizaine d'autres monographies, sur des quartiers urbains de la banlieue parisienne et de la province, avec un fil conducteur d'analyse commun : quelles sont les politiques de sécurité mises en oeuvre par les galeries marchandes, centres commerciaux ou hypermarchés, aux prises avec des problèmes de délinquances et des incivilités. Il s'agit à terme de déterminer, à partir de caractéristiques environnementales différentes, comment la grande distribution conçoit et pratique effectivement la prévention d'incivilités sur des espaces privés ouverts au public. Comment s'y concilient la raison d'être première d'un lieu de vente, avec un espace souvent vécu comme un lieu de vie.

\section{Environnement urbain et situation de crise}

En toile de fond, une cité à problèmes dans une ville de banlieue française: à la fin de l'hiver 1991, y éclate un drame. 


\section{A. Eléments de contexte}

Un quartier réputét problématique d'une ville de la banlieue parisienne est le thêâtre d'une actualité bralante depuis deux ans. L'enquête fouillée d'un service de prévention spécialisée (Sauvegarde, 1992) montre à son propos des caractéristiques hélas assez classiques:

- Une population du quartier de 7000 habitants, composée à $50 \%$ de jeunes de 0 à 25 ans. Leurs «regroupements» ${ }^{5}$ font l'objet d'incessantes polémiques et posent problème à l'ensemble des professionnels de l'action sociale.

- Ces regroupements de jeunes sont omniprésents et exercent sur chacun des membres des pressions très fortes,

- L'autonomie et la mobilité des groupes dans la cité et à son pourtour sont étendues,

- Les jeunes dont les formes de regroupement sont visibles et observables, font essentiellement partie de la communauté maghrébine. Leur omniprésence tient à des raisons historiques: les maghrébins furent la première communauté étrangère implantée au début des années 1970 dans ce quartier, après la suppression des bidonvilles d'une autre ville périphérique voisine. Elle tient également à des raisons économiques: on a affaire à des familles d'origine modeste (ouvriers et employés), précaires, qui ont des difficultés d'identité et de reconnaissance culturelle.

- Les groupes de jeunes sont essentiellement masculins. Il n'existe pas de regroupements féminins.

- Les processus d'enfermement et d'exclusion ont tendance à couper les jeunes des institutions de socialisation extérieures. Surtout, s'opère un rejet des institutions scolaires, cumulé à une absence apparente d'autorité des parents: des conflits de générations et de valeurs se laissent observer dans des faits tels que l'absence de relations entre petits et grands, la démission de certaines

4 Sur l'importance de la notion de réputation, voir Dulong et Paperman (1992) qui justifient l'inutilité de désigner nominalement les cités d problèmes étudiées - même si on les reconnaît au passage - pour éviter l'inconvénient de connoter les démonstrations, par le préjugé qu'induisent les images médiatiques forgeant les consciences des lecteurs à propos des banlieues. De la même façon, peu nous importe qu'on reconnaisse le quartier, objet de l'enquête, étant entendu que seule la logique d'une démonstration nous guide, et qu'on assume l'idée que les conclusions tirees de cette monographie ne pourraient être éventuellement transposées qu'au prix de la transparence totale des situations et des acteurs.

5 Notons que dans ce rapport, le terme de bande n'est jamais utilise, sans doute pour ne pas participer aux travers de l'imagerie médiatique ambiante qui a sérieusement tendance à rendre réels des phénomènes qui resteraient purement latents s'ils n'étaient que peu publicisés. Quoiqu'il en soit, à propos de l'objectivation de la réalité des bandes, sociologues (notamment, Dubet, 1987, Renouard, 1992, Mauger, 1993) et représentants de la police (R.G., 1990) s'opposent parfois vigoureusement. 
familles dans l'éducation de leurs enfants, la désobéissance et l'irrespect des plus petits envers les plus grands.

- Des processus de désinsertion, d'échec, d'exclusion scolaire se voient à différents niveaux des groupes d'âges. Des phénomènes de marginalisation s'accompagnent de conduites délinquantes (vandalisme, dégradations de biens publics ou privés, vol, recel, incarcérations et suivis judiciaires), et/ou de pratiques toxicomaniaques.

- Des actes de violence répétitifs (sous imprégnation alcoolique bien souvent), deal, troc, trafic de marchandises, induisent une économie parallèle et souterraine, où des jeunes peuvent être amenés à trouver dans des actions illégales données par l'exemple de meneurs, des moyens d'existence propres à satisfaire leurs besoins.

- Les processus d'atteintes à la santé liés à la consommation de drogues sont les formes les plus avancées de l'exclusion sociale,

- Sociologiquement, loi du silence, sentiment d'insécurité, indifférence ou peur des représailles accompagnent les actes de délinquance et de marginalisation. Les délits sur le domaine public auraient tendance à prendre des formes spectaculaires (dégradations, feux de caves, incendies de voitures, agressions...) en certaines occasions. D'où, des peurs avec attitudes de repli, des comportements xénophobes, des mécanismes de défense et de protection vécus par l'ensemble de la cité...

\section{B. Moments clés d'une situation de crise}

Mardi 26 mars 1991, à la cafétéria du centre commercial voisin de la cité, $20 \mathrm{~h} \mathrm{50,} \mathrm{une} \mathrm{altercation} \mathrm{opposant} \mathrm{la} \mathrm{sécurité} \mathrm{du} \mathrm{centre} \mathrm{à} \mathrm{un} \mathrm{groupe} \mathrm{de} \mathrm{jeunes} \mathrm{dégé-}$ nère: Djamel, 18 ans, est abattu par un agent de surveillance de 27 ans, Kamel, d'un coup de fusil à pompe. Cet agent, et l'un de ses collègues, transportent la victime mourante, à l'hôpital de la préfecture la plus proche, où ils seront interpellés par la police. Placés en garde à vue par le SRPJ', ils sont interrogés dès le lendemain matin par les policiers qui ne parviennent pas à mettre la main sur l'arme du meurtre, d'où la recherche d'un troisième complice ayant eu pour rôle de faire disparaître l'arme. Entre temps, vers 22 h 30 jusqu'à minuit trente, la nouvelle de l'agression fait affluer une centaine de jeunes du quartier voisin qui, révoltés, s'en prennent alors aux vitres de la cafétéria, et laisseront plus tard un parking dévasté non sans avoir incendié quelques voitures au passage, avant de se disperser. Les rumeurs les plus folles se sont répandues comme une traînée de poudre, laissant croire que les vigiles sont allés jeter le corps de la victime sur le pont du fleuve voisin. Ce début d'émeute s'accompagne de la volonté de lyncher les auteurs de

6 Service Régional de Police Judiciaire. 
l'agression, dont on pense que la plupart se sont réfugiés à l'intérieur du supermarché. Le directeur départemental des polices urbaines, dont chacun saluera par la suite le courage et le sang-froid, parlemente durant deux heures avec certains porte-parole des jeunes, responsables d'une maison de quartier; ensemble, ils réussissent à calmer les esprits échauffés. A 2 heures du matin enfin, une compagnie de $\mathrm{CRS}^{7}$ évacue les sept agents de sécurité retranchés à l'étage du centre.

L'affaire aurait pu en rester là, si le maire de la commune n'était venu entre temps fort malencontreusement attiser sur place le conflit, réclamant à corps et à cris aux policiers la dispersion des voyous, accusant la police de tergiversations, l'usage de la force publique, qui ne lui appartient pas à son grand désespoir, constituant le seul langage et la seule réponse adéquate pour faire cesser selon lui, la révolte des casseurs et émeutiers arabes.

Le premier vigile écroué sera inculpé d'homicide volontaire et port d'arme prohibé, le deuxième de complicité, le troisième, de transport d'arme prohibée, et de dissimulation d'indices. Ces deux derniers seront remis en liberté à l'automne 1991, le meurtrier quant à lui, sera remis en liberté sous contrôle judiciaire par la chambre d'accusation de la cour d'appel un an plus tard, en octobre 19928. Or, cette décision judiciaire de remise en liberté, assortie d'une interdiction de séjour dans le département du drame et d'un autre limitrophe, préoccupe les autorités préfectorales, tant le contexte ne leur semble pas encore vraiment apaisé aujourd'hui. Si la comparaison n'est pas forcément pertinente, il n'en demeure pas moins vrai que garantes de l'ordre public, les autorités restent d'autant plus en état d'alerte qu'elles gardent le souvenir des émeutes de Los Angeles du printemps 19929.

Mais les violences reprennent dès le jeudi soir 28 mars dans le quartier de la cité, où plusieurs dizaines de jeunes assaillent les CRS stationnés aux alentours du centre commercial qu'ils quadrillent; des pierres et des boules de pétanque sont lancées contre le supermarché. Un incendie éclate dans un immeuble de la ville, près de la gare. Neuf personnes parmi les émeutiers sont interpellées et placées en garde à vue; quatre sont remises en liberté le lendemain, dont semble-t-il, trois agresseurs présumés du premier adjoint au maire entre temps malmené, blessé au visage pour s'être opposé physiquement dans la ville à une scène de pillage commise par des jeunes. L'agresseur direct, 21 ans, retrouvé, est inculpé de violences à magistrat, et écroué le 2 avril. Les circonstances de cette affaire demeurent assez obscures, en tout cas les versions sont là encore contradictoires... Le directeur des polices urbaines déclare le samedi: maintenant, il est clair que nous avons affaire à la frange délinquante de la Cité. Autant nous nous sommes montrés conciliants depuis mardi soir, autant aujourd'hui nous réagissons avec énergie face à des voyous qui font preuve d'une certaine organisation dans leur manière de harceler la police. L'échauffourée a fait cinq blessés légers parmi les policiers, un blessé parmi les jeunes.

7 Compagnies républicaines de sécurité: unités de police spécialisées dans le maintien de l'ordre.

8 L'affaire n'a pas encore été jugée, au moment où nous écrivons.

- Liées, on s'en souvient, à l'annonce du verdict d'acquittement de policiers dont une caméra indiscrète avait filmé les méthodes d'action retransmises à l'univers entier. 
Dès le vendredi, le responsable du plus puissant syndicat de policiers (FASP) exhorte de son côté solennellement par voie de presse les pouvoirs publics à mettre en place une véritable politique de la ville, et à faire toute la lumière sur la société de gardiennage, à l'origine du meurtre. Il rappelle qu'un rapport de police daté du 9 avril 1990, faisait état d'une affaire où un enfant de 11 ans, accusé d'un vol à la tire dans le même type de supermarché d'une autre banlieue, avait été menacé par le vigile de la même société de gardiennage avec un révolver de 9 mm: je vais te tirer une balle dans la tête. Cette affaire avait été classée sans suite au parquet. Le vigile, muté ailleurs pendant quelques mois, était revenu exercer ses fonctions au même endroit un peu plus tard. Aucune disposition n'a été prise. Il est scandaleux que cette société n'ait pas fait l'objet d'un plus grand contrôle, ajoute le syndicaliste policier qui demande au gouvernement de se prononcer sur les activités des sociétés de gardiennage et d'adopter une attitude ferme à leur égard. Un arrêté de suspension provisoire immédiatement exécutoire entériné par le ministre de l'Intérieur sera pris le 5 avril, à l'encontre de la société de gardiennage, ce qui rend ses activités définitivement illégales.

Le samedi après-midi $\mathbf{3 0}$ mars, une marche silencieuse de la population locale est organisée dans la ville. Certains réclament la démission du maire pour ses attitudes provocatrices, mais la plupart des habitants sont là pour protester contre la décision du centre commercial qui a réouvert ses portes dès le lendemain du drame, ce qui est analysé comme une autre forme de provocation, alors que la cafétéria, elle, restera fermée durant une semaine.

Très rapidement, le ministre de la Ville lance une invitation aux élus, fonctionnaires, représentants des associations et des jeunes, afin de se réunir à la préfecture. Un sous-préfet à la ville, parmi les treize créés 15 jours plus tôt, a déjà rencontré à la fois le maire, mais aussi le chef du projet DSQ dont fait partie la ville où se trouve le quartier. Une convention avait en effet été signée six mois auparavant, le 2 octobre 1990, mais ce quartier n'avait pas été à l'époque considéré, parmi douze quartiers du département faisant l'objet de cette procédure, comme étant le plus difficile. La répercussion médiatique du drame ayant évidemment pris une ampleur nationale, le premier ministre en personne annonce, dès le 4 avril, deux mesures: l'accélération des procédures de naturalisation, et le prochain dépôt, devant le Parlement, d'un texte préparé depuis 1989, dit de la sécurité intérieure, visant à clarifier les compétences respectives de la police nationale, de la gendarmerie et des douanes, des polices municipales et des sociétés privées de gardiennage. Le ministre de la Ville joue quant à lui les médiateurs. Il dialogue le même jour à la préfecture, avec quarante personnes, hauts fonctionnaires, élus locaux, représentants de treize services départementaux, animateurs d'associations et, fait notable, avec huit jeunes du quartier, pour la plupart d'origine maghrébine. Ils sont fortement incités à cette occasion à créer une association différente de celle qui existe sur la ville, une association ciblée au quartier, de façon pour l'administration à disposer d'une instance d'interlocuteurs capables d'exprimer les besoins spécifiques des jeunes de ce quartier-ci. Le maire, de son côté, apparemment satisfait des engagements pris par les pouvoirs publics lors de cette réunion, fait voter par son conseil municipal réuni le 7 
mai, une participation de 3 millions de francs pour enrichir le programme général DSQ et l'opération de réhabilitation du bâti de la cité. La grande réunion prévue par le préfet se tiendra durant six heures à la préfecture le 13 mai suivant. Elle rassemble tous les partenaires susceptibles d'intervenir dans le développement social de la ville et du quartier en particulier (associations de jeunes, Etat, villes, département, société HLM, locataires, commerçants) afin de prendre des mesures concrètes destinées à recréer un tissu social dans cet ensemble.

Durant deux mois, la tension sur le site demeure encore très vive. La presse signale de nombreux incidents (bagarres, violences) opposant des jeunes et des vigiles dans et autour de certains hypermarchés des banlieues parisiennes. On retiendra tout particulièrement l'un de ces incidents survenu au moment de la reconstitution du meurtre par le juge d'instruction chargé de l'enquête, dans la soirée du 10 avril, où des cocktails molotov et des pierres sont lancés sur les CRS qui ripostent en lançant des grenades lacrymogènes (la présence assidue de la télévision n'étant pas pour rien dans cette continuelle stratégie de la tension). Au commissariat de police, durant ces deux mois, quarante personnes ont été interpellées, trente gardées à vue, et huit finalement écrouées.

Deux ou trois nouvelles sociétés de gardiennage vont se succéder au supermarché jusqu'en juin, et régulièrement échouer. La matraque et les chiens sont les seules ripostes opposées à des jeunes meneurs révoltés qui incitent les autres au pillage, non sans rechercher la fermeture définitive du centre devant lequel est tombé leur camarade. Des stratégies de médiation de la direction du magasin avec les parents échouent, mais également de maladroites actions de sponsoring d'activités en direction des jeunes. On leur donne des vêtements de sport, on loue des gradins pour des matches de football, etc. en attendant les ressources des classiques opérations anti été-chaud. Le centre commercial tente d'inventer des formules de prévention dans la précipitation: des vigiles loués en catastrophe désertent rapidement le centre. Les dirigeants du supermarché acceptent temporairement l'idée que pour calmer les esprits, il faut consentir à certains actes de vol provoqués par la haine ou la vengeance, plutôt que de pratiquer systématiquement des interpellations en attendant les forces de police. En deux mois, $20 \%$ de la clientèle a déserté le supermarché, et est considérée comme définitivement perdue. Ce n'est qu'au prix des efforts conjugués des autorités préfectorales et de certaines associations que le centre ne fermera pas. Les autorités savent en effet que mise à exécution, cette fermeture constituerait une catastrophe économique: accentuation du chômage, les salariés de la grande surface étant licenciés, désertion de vingt-cinq petits commerces vivant dans la dépendance du supermarché, mais surtout, marginalisation définitive des petites gens du quartier ayant par ailleurs les plus grandes difficultés pour aller s'approvisionner ailleurs...

\section{Changer les pratiques de l'ordre sur l'espace commercial}

Rien ne prédisposait jusqu'à présent deux jeunes champions d'arts martiaux (boxe thai et karaté), français de souche maghrébine, par ailleurs éducateurs 
sportifs, à se lancer, avec la pugnacité d'un gestionnaire de grande surface inventif, dans la mise en ouvre d'un pari audacieux capable de faire baisser la pression au cour de la plaie. L'innovation dont nous allons rendre compte se donne à lire sur trois plans: une conviction qui guide des actes (la violence des jeunes peut être canalisée); des pratiques de régulation originales (rompre avec la fatalité de l'absence de communication entre parents et jeunes); une polarisation sur des résultats mesurables, comme critères d'efficacité et de légitimité sociale.

\section{A. Naissance et mise au point d'un projet de gardiennage nouvelle manière}

L'alternative proposée et négociée intervient dans les mois qui suivent le drame, et devient véritablement opérationnelle à la rentrée 1991.

a) Canaliser le potentiel de violence des jeunes par la pratique du sport.

L'expérience de prévention originale dans un contexte aussi perturbé ne fut possible que grâce à la conjonction de trois facteurs:

- Un capital de légitimité antérieurement acquis par les futurs dirigeants de la société de gardiennage. Ce sont des champions reconnus dans leur spécialité, éducateurs sportifs, dont l'un est de surcroît un ancien agent contractuel du GIPR ${ }^{10}$. Tous deux promeuvent une philosophie positive du sport en direction des jeunes du quartier en difficulté. Leur bonne connaissance des jeunes et de leurs parents est telle qu'au bout d'une année, l'équipe de gardiennage dans son ensemble parvient à nommer par leurs prénoms et à connaître la nature spécifique des problèmes de chacun des quatre cents jeunes qui flânent de temps à autre dans le supermarché.

- L'état d'urgence où la direction du supermarché est acculée en juin 1991, la conduit à vouloir régler une bonne fois pour toutes et à n'importe quel prix, les problèmes d'insécurité. Elle participe alors à la constitution d'une société de gardiennage beur ${ }^{11}$ ayant carte blanche pour expérimenter des méthodes d'action alternatives avant tout basées sur le dialogue, la courtoisie et la fermeté.

- Le pari des autorités préfectorales, très rapidement convaincues du bienfondé du projet par la direction du centre, est de faciliter la constitution juridique de la société, quitte à amortir les sources de conflits liés à des pratiques inhabituelles pouvant indisposer la police. A cet égard, police et gendarmerie ont fait l'objet de larges explications préalables quant au projet, les premiers restant sur la défensive, les seconds se montrant d'emblée plus enthousiastes.

10 Brigade d'intervention et de protection des réseaux. C'est un corps de police urbaine spécialement affecté à des operations de contrôle dans le métro (RATP) et le réseau express régional (SNCF) pour pallier les défaillances des effectifs de la police.

"Arabe, à l'envers (verlan). Désigne par commodité les jeunes de la deuxième (voire troisième) génération de l'immigration maghrébine, pour la plupart nés en France, de nationalité française en vertu du droit du sol (jus soli). 
Or, dans les premiers mois qui voient vivre le début de l'expérience, (et notamment ce moment délicat entre tous, celui où les jeunes ayant bénéficié des fameuses «opérations anti-été chaud» retournent à leur cité ennuyeuse), les occasions de conflits entre la police et les vigiles restent nombreuses.

- En pratique, la solution a consisté à mettre en place deux équipes de douze surveillants, beurs pour vingt d'entre eux ${ }^{12}$, qui ont été sélectionnés de manière très habile. En effet, on a recruté, ou bien les plus méritants des élèves des éducateurs sportifs, ceux qui font montre d'une ferme volonté d'intégration, de leur envie de sortir de la galère ${ }^{13}$ et du chômage par les moyens légaux disponibles. Ou bien, non sans un certain sens avisé de la tactique, en faisant appel à certains des jeunes ou frères aînés d'adolescents à problèmes, perçus comme fortement perturbateurs. Cette stratégie semble payante puisqu'elle rend beaucoup plus difficile la pratique du vol à l'étalage et les occasions de chercher la bagarre. Avec cette méthode, il leur devient impossible de voler, ou d'embêter les gens. Pourquoi? Parce qu'ils ne peuvent pas vivre avec l'image d'un grand frère qui les voit comme des petits voleurs, qui ne leur renvoie pas d'attitude de tolérance face au vol, alors que les délits sont souvent considérés par eux comme une pratique de rebellion légitime à la condition faite aux exclus de la société à laquelle ils ont le sentiment d'appartenir. La meilleure preuve que nous visions juste, c'est qu'au moment des embauches, on a eu des chantages incroyables de la part des plus jeunes. Ils nous racontaient que leurs «aînés» n'étaient pas propres, etc. En fait, ne pouvant pas leur manquer de respect, ni leur cracher dessus, ils savaient bien qu'ils n'allaient plus pouvoir faire n'importe quoi, commente l'un des dirigeants de la société de surveillance...

b) Agir d'abord en direction des parents, plutôt qu'en direction de la police.

Concrètement, en faisant un travail pédagogique moralisateur constant auprès des parents, maintes situations-problèmes ont pu être désamorcées avec une relative douceur et sans drame, qui n'avaient pu trouver de solution à cause d'une communication dégradée entre parents primo-immigrants et adolescents nés en France. Phénomène typique, les parents maghrébins entretiennent un amour immodéré pour leurs enfants, mais c'est un amour mythique au point de les rendre aveugles et sourds aux conséquences de leur désoeuvrement réel. Ils les imaginent à l'école ou à faire du sport. Or, quand les jeunes, dans leurs

12 Sur l'une de nos relances, le directeur du supermarche, pragmatique, explique: moi bien sar, je souhaiterais un mixte ethnique dans l'équipe de sécurité. Car quant la proportion des maghrébins devient trop dense, ga peut déraper... Mais aujourd'hui que les tensions sont encore fortes, il ne faut pas se leurrer: un maghrébin dialogue plus facilement avec un maghrébin. Il se trouve qu'il est plus écouté qu'un Frangais de souche...

13 Image évocatrice de l'identité de la vie déscuuvré que se donnent des jeunes dans les banlieues, sans projet d'avenir du fait du chómage et de la precarité de leur condition. Ce terme est devenu le titre de l'ouvrage d'un sociologue, François Dubet (1987). 
moments de flânerie, créent des occasions de conflits, volent à l'étalage du supermarché, agressent verbalement sinon physiquement les clients, les parents ont tendance à ne pas y croire.

Aussi, sont-ils fortement incités à venir ouvrir les yeux sur certaines pratiques de leurs enfants. On leur montre à cette intention les films pris par les caméras de vidéo-surveillance omniprésentes dans le supermarché et la galerie marchande. C'est ainsi qu'en une année, une centaine de familles convoquées s'est rendue discrètement dans le local de surveillance du supermarché, ont eu les yeux dessillés, et ont da commencer à modifier leurs schémas de croyances, envisager de trouver des solutions avec l'aide du secteur associatif et sportif, etc. Au besoin, l'encadrement des agents de surveillance n'hésite-til pas à se rendre dans les familles, dès lors qu'éclate un problème sérieux, qui demande à être réglé de toute urgence. On n'hésite pas à aller voir le père à son retour du travail, même à deux heures du matin. Parler le langage des parents, voire les idiomes d'origine, se mouler dans leur imaginaire, se mettre à la portée de leurs difficultés et de leurs incompréhensions, relève en vérité d'une stratégie de travail social qui table indirectement sur une reprise en main (négociée) des enfants et des adolescents par leurs parents. Si ces derniers sont souvent considérés comme ayant trop facilement démissionné dans le domaine éducatif, c'est qu'en général, on ne leur a pas donné les moyens de comprendre les déchirements et l'écartèlement que vivent leurs adolescents nés en France.

Le bénéfice secondaire de telles opérations vient de ce qu'au besoin, les marchandises volées pourront être restituées par les parents, leur honneur ayant été mis à mal par la «convocation». On nourrit de la sorte l'espoir que les jeunes ne puissent supporter très longtemps l'idée que leurs parents déçus par leurs comportements, se sentant obligés de réparer l'honneur familial, voire l'honneur communautaire bafoué, cherchent à faire restituer ou faire rembourser les objets volés par les jeunes. On table sur l'idée d'une reprise en main des enfants à cette occasion... Il semble qu'à part deux ou trois familles ayant refusé de collaborer, cette stratégie de la dissuasion ait été particulièrement intelligente sur le plan de l'efficacité. Même le commissaire de police reconnaît volontiers que cette méthode de régulation des problèmes envers les 12/16 ans aurait commencé à porter ses fruits.

\section{c) Sur l'espace commercial: sécurité parpaing ou sécurité éponge ${ }^{14}$ ?}

La sécurité parpaing est la sécurité jugée bornée, répondant aux provocations ou aux délits des jeunes par de la riposte musclée, de la castagne, des sévices plus ou moins raffinés, de la séquestration arbitraire. C'est une image destinée à montrer qu'à se renvoyer la balle, l'inévitable ascension aux extrêmes provoquera ce

\footnotetext{
14 Nous respectons ces deux images très évocatrices, utilisées par les promoteurs de l'expérience: elles leur tiennent lieu de définition de leur action en même temps que de mise à distance pratique de celles qu'ils condamnent.
} 
que l'on sait. Contre cette méthode dépourvue d'imagination mais assez répandue, on oppose une philosophie d'action baptisée sécurité éponge. Elle consiste à canaliser en permanence déviances et violences possibles dans l'espace commercial et la zone du parking attenant, en les absorbant de facon à les faire dériver vers des solutions de compromis, où l'art de la négociation tient lieu de politique constante. On discute, moralise, essaie de trouver des solutions pour chaque cas particulier du jeune à problème, pour peu qu'on le ressente implicitement en état de demande. Surtout, on tente de le déconnecter de l'emprise de son leader, s'il appartient à une bande plus ou moins formalisée. Dans les situations extrêmes, les agents parviennent généralement à bout de toute violence physique par les méthodes d'autodéfense douces, auxquelles ils sont par définition entraînés. Au pire, il arrive que le sujet à problème puisse être physiquement immobilisé, ce qui ne veut pas dire qu'il soit définitivement neutralisé.

Une question théorico-pratique se pose à ce sujet: faut-il se consacrer aux sujets à risques mais récupérables ou mettre plutôt l'accent sur les sujets à problèmes réputés déjà irrécupérables ${ }^{15}$ ? Ce débat agite les dirigeants de la société de gardiennage au point même de susciter parfois des désaccords entre l'administrateur et l'exploitant. Quoiqu'il en soit, des expériences de prise en charge de sujets à problèmes ont apparemment réussi à faire changer les comportements. Ainsi, lors de galas et de compétitions sportives où l'une des tactiques de mise en confiance consiste à pratiquer des transactions avec les intéressés, on assiste à des rituels de mises à l'épreuve, les expériences pouvant se poursuivre si les résultats s'avèrent concluants: on vous fait entrer gratuitement puisque vous n'avez pas les moyens de payer votre place, mais on doit pouvoir compter sur vous, en cas d'incidents ou de problèmes... On fait de surcroît le pari que le caractère passif de la prise en charge du jeune au départ se transformera avec le temps en activisme de responsabilisation. Et de fait, certains des jeunes les plus difficiles paraissent entrés dans un cycle de responsabilisation passant par la découverte de leur utilité dans des rôles sociaux qui leur sont adéquats: jouant les huissiers dans de telles enceintes, ayant pour mission de placer les spectateurs sur les gradins, ils deviennent d'efficaces animateurs de supporteurs, etc.

Quant aux méthodes employées à l'égard des têtes brâlées réputées irrécupérables, pour qui le vol à l'étalage ne constitue qu'une des activités illégales de recel et de trafic satisfaisant des pratiques toxicomaniaques rampantes, elles sont relativement simples. Lorsque toutes les autres méthodes de prévention ont échoué, un autre deal - en accord notamment avec la direction du supermarché -, finit par s'instituer avec la gendarmerie proche de la cité, ou le SRPJ de la circonscription avec lequel s'est instaurée une bonne coordination, pour régler le cas. Il s'agit d'apporter à ces organes de police judiciaire un nombre suffisant d'éléments de preuves destinées à faciliter une capture en flagrant délit, de façon à débarrasser la communauté de l'élément gangrené qui l'affecte. Indices et

15 Selon les terminologies indigènes spontanément utilisées. 
preuves accumulés dans le supermarché par la mémoire archivée des caméras sont de la plus grande utilité pour aider à l'identification d'un délinquant par ailleurs soupçonné et activement recherché par la police...

On le voit, la cohérence du projet et les pratiques qu'elles induisent sont telles qu'elles ont fini par porter leurs fruits au bout d'une année. S'il existe bien encore dix ou quinze irréductibles dans le quartier, au moins ne parviennent-ils plus à entraîner dans leur sillage des jeunes qui, depuis le meurtre, ne communiquaient plus que dans la haine de la police, des autorités publiques et des vigiles du supermarché... Haine et rage d'autant plus vives que fortement alimentées par des effets d'admiration et d'emprise à l'egard de certains leaders directs qui manipulent ces sentiments à leur profit, soit pour s'enrichir directement comme dans des logiques maffieuses, soit pour saper tous les efforts d'intégration entrepris en détournant les énergies à des fins politico-religieuses... possiblement subversives ${ }^{16}$.

d) Déstabilisation et renouveau du travail social, en guise de bilan provisoire. Pour imposer cette pédagogie quotidiennement inventive de la carotte et du bâton, la nouvelle équipe de sécurité du supermarché a dô prendre garde au départ à son image, c'est-à-dire à ne pas apparaître auprès des jeunes comme une auxiliaire de la police officielle, et pour dire bref, comme autant de balances, trâtres, pourris, vendus, qualificatifs dont elle fut largement affublée au départ... Comme toute expérience qui réussit à changer, fut-ce symboliquement, quelque chose au sein d'une situation bloquée, il est intéressant d'observer que les critiques n'ont pas manqué de fuser de côtés d'ordinaire diamétralement opposés:

- Du côté de la police, on aurait plutôt tendance à penser l'expérience dans les catégories analogues à celles des petits commercants de la galerie marchande, c'est-à-dire plutôt selon les logiques de l'amalgame ethnique: des beurs imposent à tout le monde des méthodes pires que le mal qu'elles sont censées combattre; la tolérance au vol y étant érigée en système avec l'accord de la direction du centre, ne peut s'installer en pratique qu'un système de collusion objective de pratiques illégales. Encore plus difficile dès lors d'inverser la tendance et de savoir sur quoi pareille expérience pourrait déboucher....

- Du côté travail social pro-jeunes, d'idéologie ouvertement anti-répressive, les réflexions peuvent quant à elles se résumer en deux directions:

- ou bien se fait sentir une tendance à assimiler ce genre d'expérience de gardiennage à une nouvelle mouture soft de répression, comme si les vigiles, quelle que soit la couleur de leur peau, n'étaient pas par définition les alliés objectifs des flics...

- ou bien la tendance à contester l'expérience pérennise l'idée que les beurs ne seraient fatalement destinés qu'à remplir des fonctions sociales de

16 Sur ce point, voir Bernard, 1992. 
parias. Les vigiles beurs seraient disqualifiés parce qu'ils accepteraient d'assumer de nouvelles formes de balayage ou de nettoyage: réprimer leurs semblables, participer aux processus d'exclusion dont ils seraient partie intégrante sans s'en apercevoir, puisqu'une certaine idéologie des affaires aujourd'hui valorisée les aurait précipités dans le panneau de la récupération par l'intégration.

Un tel front d'hostilités prouve au moins qu'on est bien en présence d'une expérience déstabilisante, puisqu'elle provoque une convergence de malaises sinon de refus, venus tant de la part des policiers que des travailleurs sociaux (animateurs socio-culturels) que tout oppose par ailleurs ${ }^{17}$. La critique idoine formulée de part et d'autre s'exprime dans l'argumentaire selon lequel le laxisme à l'égard du vol conduirait à une véritable catastrophe sociale: les jeunes éléments sains de la communauté, peut-être plus enclins à pratiquer le calcul coât/bénéfice de la conduite délictueuse, pourraient se mettre à imiter les pratiques de vol, de saccage et de pillage, s'ils sont suffisamment bien travaillés par certains de leurs leaders à propos de la croyance qu'aucune répression ne s'exerçant, ces pratiques seraient admissibles, voire idéologiquement légitimes. De surcroît, les phénomènes parallèles de justice privée deviendraient de moins en moins contrôlables.

Par delà ces rationalisations réprobatrices, observons surtout que pour les besoins de la mise à distance d'avec l'expérience, la même argumentation, fondée sur une théorie criminologique spontanée de la délinquance situationnelle (Killias, 1991, 287-331), est tenue par des acteurs qui n'ont évidemment pas l'habitude de lire les phénomènes de délinquance urbaine selon les mêmes schémas causalistes... On sait que grosso modo, les travailleurs sociaux préfèrent mettre l'accent sur les «causes» sociales externes aux conduites délinquantes (pauvreté, misère, chômage...), tandis que les policiers préfèrent insister sur les "causalités» internes à la défaillance de la socialisation de ceux qui seront les délinquants de demain.

C'est que toute innovation sociale provoquée par des acteurs entrant dans un champ et un espace qui n'a pas été défini par eux est toujours vécue comme une menace pour l'identité des acteurs qui se partageaient jusque là plus ou moins

17 Deux témoignages emblèmes de visions du monde radicalement opposées, presque repliées sur elles-mêmes... se donnent à voir dans ces morceaux d'entretien:

Un animateur beur en poste sur la cité: Ce qui est pour moi insupportable, ce sont ces éternels controles d'identité et les mises en boftes qui s'ensuivent... Par exemple, apres avoir vérifí mes papiers, on me demande dans le métro: *Tu fais quel boulot? * Réponse: *Je suis éducateur-animateur»; «Ah oui, tu défends les délinquants, quoi!...* *Quand cela va trop loin, je demande dappeler le sous-prefet, que je connais personnellement." Réaction invariable: «Ah? Excusez-nous, Monsieur, nous ne savions pas!...

Le commissaire, à propos de l'image médiatique des fameux kquartiers interdits» à la police: De toute façon, quoique vous disiez ou fassiez, à propos des maghrébins, les blancs vous reprocheront toujours: «Vous n'osez pas y aller parce que vous avez peur des arabes qui y font la loi. * Les maghrébins vous diront: «Vous n'allez que là, parce que vous présumez qu'il y a plus de delinquants dans ces quartiers, et vous le croyez parce que vous êtes racistes.» 
implicitement le travail social de régulation par la répression ou la prévention. On peut penser qu'un compromis implicite s'était organisé entre eux autour de la dénonciation et du rejet de la classique solution des gros bras qui ne constituerait qu'une impasse. Or, par son nouvel empiètement sur des attributions et modi operandi déjà éprouvés, cette expérience à l'interface du gardiennage et du travail social, déstabilise effectivement les schèmes de perception et logiques d'intervention des agents traditionnels du contrôle social. Ils risquent de voir, au moins en ce qui concerne les travailleurs sociaux, leurs clientèles se détourner de leurs projets, ce qui pourrait vouloir dire à terme, une situation les obligeant à remettre sérieusement en cause leur propre statut social. En réalité, cette expérience n'a jamais prétendu envenimer les rapports avec les jeunes de la cité, ni nuire aux relations nécessairement synchrones avec les objectifs d'intégration visés par les autres travailleurs sociaux de la cité. Toutefois, en prenant autrement en charge les problèmes spécifiques des populations jeunes (catégories moins ciblées que les clientèles habituelles des travailleurs sociaux), et en renvoyant très sélectivement certains de leurs membres aux autorités policières et judiciaires quand la nécessité s'en fait sentir, cette équipe de sécurité irrite, exaspère, bref, perturbe le statu quo. Parce qu'elle agit selon des objectifs et des critères implicites, généralement mal compris à l'extérieur du site... Ces critères implicites sont en revanche tout à fait explicites pour ceux qui financent l'expérience.

\section{B. Le prix d'une sécurité efficace?}

C'est la question essentielle: si le succès des actions «d'intégration» des jeunes par le sport restent difficilement mesurables dans l'absolu, (et restent même fort sujettes à caution pour plusieurs catégories d'acteurs), à l'intérieur du supermarché, le calcul du coût de la sécurité mise en place reste quelque chose de palpable et de parfaitement évaluable.

a) Calculs à plus long terme, contre réticences immédiates...

Doser stratégiquement une certaine marge d'acceptation du vol sur des produits symboliques tels que vêtements, chaussures de sport, tous articles dernier cri dont les jeunes se montrent en général friands, pour qui paraître à la mode est sacré... c'est évidemment l'une des politiques qui suscite le plus de réticences et d'hostilités. On se scandalise de cette pratique, comme si elle avait toujours été conçue par ses promoteurs de façon dogmatique et non évolutive; ce qui permet à peu de frais à ses détracteurs de mieux dévider des rancoeurs plus générales.

- D'abord, de la plupart des petits commerçants de la galerie marchande, qui préfèrent les classiques méthodes parpaing des vigiles, armoires à glace ou autres gros bras. Non seulement parce qu'elles pourraient compenser des pulsions agressives vécues en quelque sorte par procuration, mais surtout, parce que pour eux, l'ordre doit être restauré de façon visible et palpable. Il ne saurait l'être qu'à partir du moment où l'on peut constater qu'il s'imprime sur le 
corps des mauvais sujets, les sujets menaçants. Pour les petits commercants, se sentir en état d'alerte ou de victimation par la faute des jeunes, les conduit à deux attitudes non antithétiques: selon les circonstances, ils peuvent se montrer bienveillants, c'est-à-dire interventionnistes dans le social (Roché, 1990, 3), s'ils n'ont jamais été victimes. Dans les autres cas, ils désirent la présence répressive de la police. A défaut, celle de vigiles musclés dirons-nous dans le cas présent. Car ici, leurs repères sont brouillés par une expérience qui leur demeure mal compréhensible... Le directeur et gestionnaire de la sécurité du supermarché ne partage pas les valeurs et options de ceux dont il protège objectivement le petit commerce. Dans son discours, la protection évoquée peut être décrite selon deux modalités: une aptitude à trouver des solutions ayant permis d'éviter la fermeture définitive de la galerie marchande (la grande surface sauve donc la mise aux petits commercants); la contrepartie implicite, c'est de la part de ces derniers, d'accepter bon gré mal gré ces méthodes originales de réduction de la tension sociale. Au départ, les petits commercants du site furent très réticents à admettre le bien-fondé de cette stratégie expérimentale, même s'ils furent les bénéficiaires directs de ses retombées principales, ce qu'ils ont progressivement fini par admettre.

- Ensuite des associations de quartier qui, mal dotées en moyens et dont les subsides tombent comme par enchantement à certains moments de crise, voient leurs efforts éducatifs de longue haleine mal payés de retour, défiés par ce qu'ils perçoivent comme des «concurrents» déterminés. Les éducateurs du site ont tendance à dénoncer les sports d'initiation pratiqués comme une possible école de violence, mais, plus important, persiste chez eux la propension à stigmatiser les pratiques de collaboration des vigiles avec la police ou la gendarmerie. Les jeunes seraient au fond pris en otage, à seule fin affirme-t-on de façon plus ou moins nuancée, de satisfaire la répression. On ne pourrait être à la fois au four et au moulin: dans ce domaine, il conviendrait impérativement de choisir son camp. Si les «acteurs sociaux» ont toujours de bons exemples à donner pour illustrer ce qu'ils avancent, on perçoit néanmoins chez ces derniers tout le drame d'un travail social mis en question, qui parvient de moins en moins bien à retrouver ses marques, et plein d'anxiété face à ce qu'il ressent confusément comme les coups de butoir de nouveaux travailleurs sociaux concurrents (Faget, 1992). Des gestionnaires originaux capables d'inventer des projets inédits, de trouver des sources de financement et de les gérer, et même de s'en faire les ambassadeurs ou propagandistes (CNFEPJJ, 1992).

- Enfin de la police qui, par un curieux renversement de situation, aurait tendance à contester l'idée d'avoir à surveiller continuellement le site commercial en fonction des moyens publics limités dont elle disposerait, et surtout au nom de l'intérêt général dont elle serait le seul représentant. L'intérêt général commande la mission généraliste, c'est-à-dire d'apporter des réponses à toutes les demandes sociales, sans sollicitude particulière pour certaines clien- 
tèles au détriment des autres. Or, face à cette expérience, la police en patrouille ressent paradoxalement très mal le caractère indésirable de sa présence dans la galerie marchande ou à ses abords, quand elle est amenée à s'y rendre. Du côté du service de sécurité, on réagit à la mise en cause en arguant qu'en effet, la présence de la police en tenue et armée insécuriserait la clientèle, plutôt que de la rassurer ${ }^{18}$. Derrière les arguments de la police où se mêlent l'idéologie syndicale générale et les constats amers de la vie quotidienne, se laisse facilement percevoir l'impression insupportable qu'elle pourrait devenir l'instrument, sinon passer pour l'auxiliaire du service de sécurité du supermarché. Cette menace n'est pas vraiment sérieuse, car il est évident que le centre est toujours à la merci par exemple d'une rumeur, d'un quelconque geste politique maladroit, d'une bavure qui peuvent faire dégénérer la situation, le recours à la force publique sera alors une question de survie... La situation peut en effet redevenir à tout moment explosive, et les forces de l'ordre être mobilisées par les autorités préfectorales pour protéger le centre commercial. Aucun agent de surveillance serait assez fou pour prétendre assurer des missions de maintien de l'ordre.

Mais en vérité ces difficultés de coordination ne proviendraient-elles pas surtout des pratiques volontaires de mise à distance ou d'évitement de la police, vue comme un partenaire plutôt inadéquat en certaines circonstances? Et cela, d'autant plus manifestement que l'habitude aurait été prise, depuis le drame, de s'adresser directement à d'autres échelons de la hiérarchie policière, en courtcircuitant précisément le commissariat de la ville?...

b) Calculs à court terme ...

Le prix de l'efficacité de la sécurité s'évalue selon la direction du supermarché, au travers de plusieurs paramètres:

D'abord, démanteler des réseaux d'écoulement de marchandises, par les employés ou manutentionnaires eux-mêmes, dont on sait qu'ils sont en général à l'origine d'une part importante de la démarque inconnue (Chevallier Baumel, Demory, 1986, 16). En l'occurrence, l'équipe de sécurité du centre aurait réussi à faire licencier une dizaine d'employés surpris en flagrant délit et à tarir une importante filière de pertes de matériels hi-fi notamment. Elle aurait également fait judicieusement déplacer des caissières soupçonnées de passer frauduleusement des marchandises à des complices...

Etre en accord avec et impulser une méthode qui sur le moyen terme fasse diminuer les tensions sur l'espace commercial, c'est savoir faire disparaittre les sources de violence qui ont fait fuir la clientèle, à certains moments de la journée (notamment en soirée, de 20 à 22 heures). Bref, c'est avant tout chercher à rega-

18 Les mains-courantes du commissariat de police attestent effectivement de procès-verbaux d'agents éconduits par la sécurité du centre commercial sous prétexte que leur présence indisposerait la clientele... 
gner la confiance des clients. C'est évidemment au nombre de clients retrouvés, au sentiment d'apaisement et de bien-être éprouvé dans le supermarché à l'occasion des multiples services rendus par les agents, que la sécurité devient rentable. Ces derniers, ayant à cœur de démontrer à la clientèle leur foncière honnêteté, leur capacité à venir en aide aux personnes âgées en cas de besoin, etc. ${ }^{19}$, sont engagés dans autant d'actions quotidiennes qui participent au gain de sécurité, donc à l'élaboration progressive de la marge bénéficiaire commerciale. Mais c'est encore le moyen le plus habile de donner au public une image réussie de l'intégration possible, rassurante et non repoussante, des jeunes beurs.

Le directeur du centre, qui se définit lui-même comme un bon flic, fait avant tout ses comptes, car c'est ce que l'on demande à un gestionnaire de grande surface, quand bien même il aurait reçu carte blanche de son directeur régional pour pratiquer l'expérience de sécurité qu'il souhaite. Il explique qu'en un an, le supermarché est passé d'une situation où l'on comptait à l'origine, $90 \%$ de prévention pour $10 \%$ de répression, à une situation où $60 \%$ des actions de sécurité concernent la prévention, contre $40 \%$ de répression. Il argumente par ailleurs sur le fait que la démarque acceptée en vol correspondait, à l'époque ayant suivi le meurtre, à la sortie quasiment tolérée de l'équivalent de 15 caddies remplis, soit Fr. 15000 . de pertes quotidiennes dues au vol pour ne pas parler de pillage..., à l'équivalent aujourd'hui d'un caddie représentant la valeur de Fr. 1500 . de marchandises. Enfin, selon lui, la récidive du vol chez les jeunes aurait considérablement diminué, car il n'y aurait plus de leaders ni de bandes de pilleurs organisées dans le secteur, la haine et les idées de vengeance tendant à s'effacer progressivement. Ces éléments lui paraissent fournir la preuve du bien-fondé d'une expérience qu'il soutient à fond depuis sa prise de fonction, et à laquelle il participe directement, étant lui-même engagé dans des actions associatives sportives auprès des jeunes. Son argumentation est imparable: des délinquants opérant seuls sont dotés d'une capacité de nuisance beaucoup plus affaiblie que lorsqu'ils opèrent en bandes organisées de pilleurs.

Au bout d'un an, l'exercice comptable ne serait pas encore équilibré, mais il a bon espoir qu'il le soit l'année suivante. Actuellement, le coût salarial de l'équipe de sécurité recrutée grève considérablement les autres investissements. Il faut payer:

- un poste d'encadrement d'un consultant pour la sécurité, cadre émargeant à plein temps dans l'entreprise (autre champion en arts martiaux, ancien garde du corps);

- le coût de la présence d'un membre fondateur de la société de gardiennage qui met la main à la pâte au besoin le jour et vit sur le site en tant que gardien la nuit;

- le coat salarial des 24 vigiles tournant en $2 \times 8$, de $9 \mathrm{~h}$ à $22 \mathrm{~h}$ par équipes de 12 , qui constitue le pôle d'investissement le plus lourd, beaucoup plus lourd que

19 Le directeur du magasin évoque: mon équipe d'agents de surveillance incorruptibles. 
la moyenne de ce qui se pratique par tranche salariale horaire dans les centres commerciaux de taille comparable.

Précisons encore que ce contrat de gardiennage est en France, l'un des plus dispendieux qui soient (indice 1,56 pour une moyenne de l). Il n'incite évidemment pas cette nouvelle race d'agents de surveillance, dont l'assistance morale ${ }^{20}$ tient lieu de mission, à vouloir casser l'image de marque de surveillants-vigiles incorruptibles qu'ils se sont forgés patiemment aux yeux de leur employeur et de la clientèle tout venant du supermarché. La cherté des prestations fournies explique l'engagement indéfectible de l'équipe de sécurité dans une mission dont tous les membres épousent à leur niveau la finalité. Elle consiste avant tout à trouver des solutions alternatives originales à la complexité des problèmes quotidiens qui se déploient sur le site. Gagner de l'argent est considéré prima facie comme accessoire par rapport aux actions de prévention socio-communautaire investies en dehors du site. Mais l'argent gagné reste un stimulant symboliquement puissant, dans la mesure où donner l'exemple d'une réussite par des voies légales, participe d'une stratégie de rupture positive vis-ă-vis de la ferveur qu'a toujours tendance à susciter chez les exclus le romantisme des révoltés agissant selon des voies illégales tout aussi valorisantes selon eux. Le musicien, le sportif, l'homme d'affaires qui deviennent des militants en mettant leurs cultures et leurs connaissances techniques au service de l'intégration ethnique, peuvent susciter bien des espoirs en montrant des voies alternatives à celles de la logique des actes désespérés.

\section{$* * *$}

Quels enseignements peut-on tirer d'une telle expérience? L'impression d'ensemble qui se dégage paraîtra sans doute prometteuse et intéressante, à ceux évidemment qui l'expérimentent et la promeuvent, mais peut-être aussi à quelques autres. Nous considérons, pour ce qui nous concerne qu'elle a au moins le mérite d'être imaginative, en concédant volontiers qu'elle reste pour l'instant assez largement atypique dans le contexte hexagonal.

Prenons quelque distance. Nous ne pensons pas que le sociologue doive se désintéresser de décrire et d'évaluer autant que faire se peut des pratiques de sécurité inventives, sous prétexte qu'elles seraient atypiques. Nous pensons que sa sociologie devrait même accomplir un pas supplémentaire en prenant éventuellement deux sortes de responsabilités: celle d'éclairer le gestionnaire politique sur ce qu'il pourrait décider au vu d'une telle expérience et de plusieurs autres quand elles seraient décrites; celle de conseiller l'essai des expériences les plus novatrices et sérieuses à l'ensemble des gestionnaires de la grande distribution commerciale, confrontés à des problèmes d'insécurité similaires, dans la mesure où elles offriraient le plus de garanties possibles.

20 Plutôt que d'assistanat social, concept formellement récusé par ses dirigeants. 
Ce ne sera pourtant pas notre propos ici. Tout simplement parce que nous ne pensons pas que toute innovation dans les pratiques de sécurisation de sites, notamment celle qui mêlerait à la fois des actions de prévention communautaire à des actions de prévention situationnelle, devrait nécessairement se voir doter d'un préjugé favorable. Les effets de telles actions pourraient même se révéler désastreux, si par excès de zèle, et à force de vouloir faire du résultat pour différents commanditaires qui ne défendraient pas les mêmes intérêts, des prestataires de service de sécurité trop autonomes étaient conduits à chercher les bonnes prises, sinon à les provoquer au besoin. Il serait facile de donner d'amples illustrations de tels risques liés au mode de fonctionnement virtuellement proactif des polices privées des centres commerciaux, en les puisant dans les informations quotidiennes de la presse, et cela, sans nul besoin de jeter l'anathème ou sacrifier à la dénonciation ${ }^{21}$ d'un complot.

A s'apercevoir que les menaces que feraient courir sur les libertés individuelles des contrôlés des agents de surveillance mal encadrés, s'avèreraient trop systématiques, on pourrait soutenir que la seule option raisonnable pour les pouvoirs publics, consisterait à interdire l'utilisation des sociétés de gardiennage et des vigiles dans les espaces commerciaux (en ce sens, Dray, 1992, 73). On peut évidemment vouloir cela. Malheureusement pour cet auteur, le principe de réalité fait que depuis une dizaine d'années, les pouvoirs publics ont fait ou dâ faire avec l'existant, ce qui veut dire qu'ils se sont convertis au fait que la police publique ne pouvait surveiller et intervenir partout (Belorgey, 1982, CNV-DSU, 1992).

Dès lors, la question qui intéresse au plus haut point aussi bien le citoyen, que le chargé des questions de sécurité sur son site (au besoin, le client d'une prestation de gardiennage), que les pouvoirs publics, demeure éminemment politique. Il nous semble qu'aucun de ces trois protagonistes ne devrait pouvoir éluder bien longtemps la réponse à ceci: à quelles conditions peut-il devenir admissible que des agents de surveillance (vigiles) soient considérés par le plus grand nombre comme des partenaires de sécurité légitimes?...

D'une certaine façon, les pouvoirs publics français ont répondu à cette question en 1983 et 1986, en faisant des entreprises de gardiennage une profession administrativement réglementée, et des agents de surveillance, des gens sans

21 Celle qui veut que les vilains maitres des temples de la consommation que sont devenus les centres commerciaux géants soient entièrement tenus pour responsables des victimations de toutes sortes qu'ils subiraient. D'aucuns affirment que l'existence même de ces centres constituerait une provocation, un véritable pousse au crime, singulièrement de la part des have not. Cette représentation qui n'est bien sor qu'un dogme gauchiste, fait allègrement fi d'au moins deux sortes de réalités: il arrive que des dirigeants de supermarché organisent le don de leur surplus à ceux qui ont faim, alors que rien, sinon la charité chrétienne, ne les y obligerait. Mais surtout, il n'est pas besoin d'etre grand clerc pour s'apercevoir que les centres commerciaux sont souvent considérés par ceux-là mêmes qui y flanent comme les seuls lieux de vie attrayants, en comparaison de leurs autres lieux de vie possibles (rues, ponts,... et tout simplement cités dortoirs, cités HLM, par exemple...). Laissons donc aux moralistes le soin de décider sur ces points s'il s'agirait là d'un effet d'aliénation lié à l'adhésion consommatoire obligée des fractions les plus dominées du peuple. 
pouvoirs supérieurs à ceux du citoyen (Ocqueteau, 1992 a,b). Or, soumise à l'épreuve des faits, on note que cette réglementation visant l'assainissement du secteur s'est avérée peu fiable en ce qu'elle n'a pas empêché véritablement la commission de graves atteintes aux libertés notamment. C'est un éternel reproche que l'on peut faire au législateur moderne: dans la mesure où il n'a pas prévu de doter l'administration d'un organe spécialisé dans le contrôle de la législation mise en place au sujet des contrôleurs privés, l'efficacité de la loi ne peut être que douteuse.

Une nouvelle adaptation du droit aux faits est donc fatalement à l'ordre du jour, puisque la réglementation n'a pas apporté les espoirs escomptés. Le sociologue du droit est dès lors raisonnablement conduit à penser qu'un nouveau défi de l'avenir consistera pour les pouvoirs publics à franchir une nouvelle étape dans le processus de reconnaissance légale du rôle des policiers privés, en dépit de la théorie du mal nécessaire de leur présence, généralement partagée par l'ensemble de la classe politique, à peu de choses près. En ce qui concerne la question de la sécurité dispensée dans le domaine de la grande distribution commerciale, on voit en effet émerger trois types de pression virtuels en direction des pouvoirs publics. Les deux premiers proviennent de lobbies de la grande distribution. La troisième pourrait provenir de citoyens éclairés:

- le remède sécuritaire maximaliste: que tous les vigiles obtiennent la qualité d'agent de police judiciaire, en tant que gardes assermentés.

- le remède plus minimaliste: favorable au statut de garde assermenté dévolu au seul chef de sécurité de l'hypermarché, à la condition que ce statut soit rendu obligatoire. Le gestionnaire de sécurité, agent d'encadrement serait ainsi tenu pour responsable des actes de son équipe de vigiles salariés ou des vigiles contractuels à qui il aurait fait appel au besoin. Ceux-ci demeureraient évidemment sans réels pouvoirs de coercition sur les citoyens, chalands, simples visiteurs ou flâneurs.

- tout cela n'exclut pas le remède prôné par le citoyen idéaliste, dont la position pourrait à plus long terme consister à tout vouloir mettre en oeuvre afin que les niveaux d'incivilités diminuent pacifiquement, pour restaurer du lien social partout où il se déliterait. De la sorte, pourrait être désamorcée une grande part de la raison d'être la plus ostensible et spectaculaire du commerce de la sécurité, à savoir un gardiennage para-policier sur les sites privés ouverts au public... A moins que le citoyen ne se satisfasse de l'apparition de gentils vigiles-éducateurs, et souhaite qu'en soit généralisée la présence pour être rassuré, tranquillisé, et partant, moins sujet à la mauvaise conscience.

Frédéric Ocqueteau

Centre de Recherches Sociologiques sur le Droit et les Institutions Pénales/CNRS

4, rue de Mondovi F - 75001 Paris 


\section{BIBLIOGRAPHIE}

ASBURY, K.E., La sécurité privée, la police publique et les grands complexes résidentiels: une étude de cas, Journal du collège canadien de police, 1990 , vol. 14, n 1 , pp. 1 29.

BELORGEY, J.M., Pré-rapport sur les réformes de la police, Paris, ministère de l'Intérieur et de la décentralisation, 1982.

BERNARD, Ph., France, terre d'Islam, Le Monde, 17 et 18 novembre 1992.

BOURDIEU, P. (éd.), La misère du monde, Paris, Seuil, 1993.

C.N.F.E.P.J.J., Les jeunes, la banlieue, les vigiles: questions sur la violence légitime, Journées de formation des 26-27 mars 1992, Vaucresson, 1992.

C.N.V., Bilan des 64 propositions du Rapport de la Commission des Maires sur la sécurité 1982-1992, Paris, C.N.V., 1992 (proposition n 48), pp. 133-134.

C.N.V., Les polices de la ville, Paris, C.N.V., 1993.

DELARUE, J.-M., Banlieues en difficulté: la relégation, Paris, Syros-Alternatives, 1992.

DEMORY, B., CHEVALIER-BEAUMEL, A., La démarque inconnue, Paris, éd. Chotard et associés, 1986.

DRAY, J., Rapport d'information sur la violence des jeunes dans les banlieues, J.O., Assemblée Nationale, Commission des affaires culturelles, familiales et sociales, $\mathrm{n}^{\circ}$ 2832, 25 juin 1992, $73 \mathrm{p}$.

DUBET, F., La galère, Paris, Fayard, 1987.

DULONG, R., PAPERMAN, P., La réputation des cités HLM, enquête sur le langage de l'insécurité, Paris, L'Harmattan, 1992.

FAGET, J., Justice et travail social, le rhizome pénal, Toulouse, éd. Erès, 1992.

HASTINGS, R., La prévention du crime: l'illusion d'un consensus, in Problèmes actuels de politique criminelle, Aix-en-Provence, Presses universitaires d'Aix-Marseille, 1993, pp. 47-69.

KILLIAS, M., Précis de criminologie, Berne, Staempfli \& C'e SA, 1991.

LAGRANGE, H., Criminalité, civilité et sociabilité: enjeux des politiques publiques, in Pré-actes du colloque organisé par le Sénat, L'engagement politique: déclin ou mutation politique, Paris, CEVIPOF, 4-6 mars 1993, pp. 763-796.

LINHART, V., De l'expérimentation à l'institutionnalisation, l'exemple de la politique de la ville, in Pré-actes du colloque organisé par le Sénat, L'engagement politique: déclin ou mutation politique, Paris, CEVIPOF, 4-6 mars 1993, pp. 797-819.

MAUGER, G., Les usages politiques du monde des bandes, in Pré-actes du colloque organisé par le Sénat, L'engagement politique: déclin ou mutation politique, Paris, CEVIPOF, 4-6 mars 1993, pp. 515-537.

OCQUETEAU, F., Gardiennage, surveillance, sécurité privée, Paris, CESDIP, 1992a, nº 56.

OCQUETEAU, F., Vigiles et policiers, concurrents ou alliés? in HAAS, P., L'Atlas économique des entreprises de sécurité 1992, Paris, éd. Technopresse-En toute sécurité, 1992b, pp. 17-23.

PERTUS, J.-J., La sécurité intérieure, Proposition de création d'une Délégation générale à la sécurité privée, Service de sécurité du groupe Casino, février 1991, doc. interne.

RENOUARD, J.-M., Les relations entre la police et les jeunes: la recherche en question, Paris, I.H.E.S.I., ronéo, 1992.

RENSEIGNEMENTS GÉNÉRAUX, Bandes de jeunes et insécurité, 22 décembre 1990, doc. interne, ronéo, $7 \mathrm{p}$. 
ROBERT, Ph., RENOUARD, J.-M., Bilan des connaissances en France, in ROBERT, Ph. (éd.), Les politiques de prévention de la délinquance à l'aune de la recherche, Paris, L'Harmattan, 1991, pp. 191-205.

ROCHE, S. (éd.), Le commerce et les jeunes, rapport d'enquête, Grenoble, C.O.D.A.S.E., ronéo, 1990.

ROCHE, S., Le sentiment d'insécurité, Paris, PUF, 1993.

SAUVEGARDE DE L'ENFANCE ET DE L'ADOLESCENCE DES X..., Rapport d'enquête Cité (X...), 15 juillet 1991-15 janvier 1992, Service de Prévention spécialisée, X..., doc. 1, ronéo, $1992,65 \mathrm{p}$.

WACQUANT, L.J.D., Banlieues françaises et ghetto noir américain. Eléments de comparaison sociologique, in WIEVIORKA, M. (éd.), Racisme et modernité, Paris, La Decouverte, 1992, pp. 263-277.

WACQUANT, L.J.D., De l'Amérique comme utopie à l'envers, in BOURDIEU, P. (éd.), La misère du monde, Paris, Seuil, 1993, pp. 169-179.

WILSON, J.Q., KELLING, G., Broken windows, The atlantic monthly, 29 mars 1982, p. 38. 